\title{
Interleukin-18 (IL-18) is equally expressed in inflammatory breast cancer and noninflammatory locally advanced breast cancer: A possible association with chemotherapy response
}

\author{
Marco Antonio Nasser Aguiar ${ }^{1}$ | Carlos Wagner S. Wanderley ${ }^{2}$ \\ Lívia Maria Soares Nobre ${ }^{2}$ ～Mateus Rolim Mendes Alencar ${ }^{2}$ \\ Maria do Perpétuo Socorro Saldanha ${ }^{3}$ | Alceu Machado Souza ${ }^{4}$ \\ Deysi Viviana Tenazoa Wong ${ }^{4}$ | Paulo Goberlânio Barros ${ }^{3,5}$

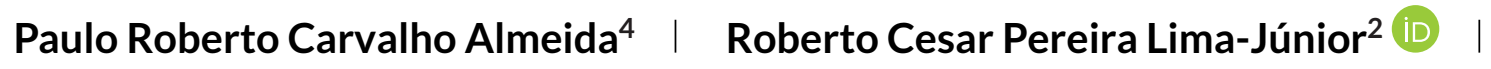

Ronaldo Albuquerque Ribeiro ${ }^{2,3}$

\author{
${ }^{1}$ Department of Surgery, Faculty of Medicine, \\ Federal University of Ceará, Brazil \\ ${ }^{2}$ Department of Physiology and Pharmacology, \\ Faculty of Medicine, Federal University of Ceará, \\ Brazil \\ ${ }^{3}$ Cancer Institute of Ceará, Brazil \\ ${ }^{4}$ Department of Pathology and Forensic \\ Medicine, Faculty of Medicine, Federal Uni- \\ versity of Ceará, Brazil \\ ${ }^{5}$ Department of Dentistry, University Center \\ Christus \\ Correspondence \\ Professor Roberto Cesar Pereira Lima Junior, \\ Departamento de Fisiologia e Farmacologia, \\ Faculdade de Medicina, Universidade Federal \\ do Ceará, Rua Cel Nunes de Melo, 1315, Rodolfo \\ Teófilo, 60430-270, Fortaleza, Ceará, Brazil. \\ Email:robertocesarpljr@gmail.com \\ Marco Antonio Nasser Aguiar and Carlos Wag- \\ ner de S. Wanderley contributed equally to the \\ development of this work. \\ Funding Information \\ Conselho Nacional de Desenvolvimento \\ Científico e Tecnológico, Grant Number: \\ 307143/2014-7, 458872/2014-8; Coordenação \\ de Aperfeiçoamento de Pessoal de Nível Supe- \\ rior, Grant Number: CAPES-PROEX 2862/2013; \\ Fundação Cearense de Apoio ao Desenvolvi- \\ mento Científico, Grant Number: PRONEX \\ PR2-0101-00054.01.00/15; de Apoio ao Desen- \\ volvimento Científico
}

\begin{abstract}
Aim: Inflammatory breast cancer (IBC) is the most aggressive form of locally advanced breast cancer. The signs of inflammation such as hyperemia and hyperthermia might suggest the possible participation of inflammatory mediators. This study investigates stromal and tumor expression of nuclear factor-kappa B (NF- $\kappa$ B) and interleukin-18 (IL-18) in samples obtained from IBC and noninflammatory locally advanced breast cancer ( $L A B C)$ and the influence of these markers on patients' prognosis.
\end{abstract}

Methods: Demographic data, tumor molecular characteristics and overall survival in both groups were also assessed. Furthermore, in this study, we evaluated the expression of IL-18 and p50 nuclear fraction of NF- $\kappa$ B by immunohistochemistry in specimens from IBC and LABC (T4b).

Results: We observed that $24.6 \%$ of women were diagnosed with IBC up to age 40 . In addition, the patients with IBC showed a lower overall survival when compared to $L A B C$. In regard to molecular markers, $\mathrm{ER}^{+}, \mathrm{C}^{-}$-erbB2 ${ }^{-}$or triple negative IBC patients showed a significantly reduced overall survival. In addition, a higher IL-18 immunostaining in stroma of IBC and LABC was observed in comparison with tumor cells, but stromal immunoexpression was similar between IBC and LABC. Besides, IL-18 positivity seemed be related with a better clinical response to neoadjuvant chemotherapy. However, NF- $\kappa$ B expression was identical in both groups.

Conclusion: The IL-18 is present in tumor stroma of IBC and LABC and seems to be associated with the complete response to neoadjuvant chemotherapy.

KEYWORDS

inflammatory breast cancer, interleukin-18 (IL-18), non-inflammatory locally advanced breast cancer, nuclear factor-kappa B (NF- $\kappa$ B)

\section{1 | INTRODUCTION}

Breast cancer is the most frequent type of cancer in women worldwide, representing $25 \%$ of all cancers diagnosed in women. ${ }^{1}$ Currently, tumor size, number of affected axillary nodes, the type and histological grade and hormonal receptors (ER, PR and C-erbB2) are widely used in clinical practice for determining prognosis and treatment response. ${ }^{2}$ However, some specific subtypes of advanced breast cancers remain associated with poor prognosis. 
The most aggressive type of locally advanced breast cancer is clinically classified as inflammatory breast carcinoma (IBC). ${ }^{3}$ Women diagnosed with IBC have an average five-year survival of $30-40 \%$, whereas patients with noninflammatory locally advanced breast cancer (LABC) the average survival in five-year is $70-80 \%{ }^{4}$

The IBC has been called "inflammatory" because the breast often shows signs of inflammation such as redness, hyperthermia and edema. ${ }^{5}$ The presence of inflammatory signs support the hypothesis that immune cells are involved in the carcinogenesis and/or tumor progression, and seem to be relevant to aggressiveness of IBC. In this context, tumor microenvironment-infiltrating cells possibly play an important role by secreting cytokines and other cellular mediators that can contribute to tumor progression. ${ }^{6}$

Nuclear factor-kappa B (NF- $\kappa$ B) is a cell-transducing transcription factor that regulates the inflammatory response, cytokine production and cell proliferation. ${ }^{7-9}$ Dysregulated activation of NF- $\kappa$ B may result in an amplified inflammatory response, altered balance of pro- and anti-apoptotic proteins and cell survival favoring tumor progression. ${ }^{10}$ Interleukin-18 (IL-18) is one of the cytokines whose expression partially involves NF- $\kappa$ B signaling. ${ }^{11}$ The IL-18 is a proinflammatory cytokine that modulates the expression of adhesion molecules, nitric oxide synthase and chemokine production. ${ }^{12}$ Interestingly, serum levels of IL-18 were found increased in breast cancer patients. ${ }^{13}$ In addition, IL-18 expression was described in tumor and surrounding tumor tissues. ${ }^{13}$ However, whether IL-18 expression accounts for a worsen prognosis in IBC and LABC breast cancer subtypes is still to be investigated.

Then, we aimed to investigate the expression of IL-18 and NF- $\kappa$ B in tumor samples of IBC and LABC and the contribution of these inflammatory markers as prognostic factors and the impact on neoadjuvant chemotherapy response.

\section{MATERIALS AND METHODS}

\subsection{Study characterization}

This was an retrospective study including a series of 75 patients diagnosed with inflammatory breast cancer (IBC) and 60 with LABC stage T4b admitted to Instituto do Cancer do Ceará and Hospital Geral de Fortaleza, Brazil, between 2012 and 2015. The inclusion criteria were (a) patients diagnosed with IBC or LABC, (b) enough sample size for immunohistochemistry studies and (c) previous immunohistochemical characterization for estrogen receptor (ER), progesterone receptor (PR) and human epidermal growth factor 2 (C-erbB2, HER2/neu). Exclusion criteria were (a) stage IV (metastatic) IBC or LABC patients, (b) insufficient tissue samples for immunohistochemistry studies and (c) lack of molecular characterization of tumor samples for ER, PR and C-erbB2 (HER2/neu).

The study was approved by the Ethics in Research Committee of Instituto do Câncer do Ceará (Protocol Number 073/2008) Hospital Geral de Fortaleza (Protocol Number 290201/12), and is accordance to the Helsinki Declaration (1983).

\subsection{Tumor samples}

The tumor samples of patients with breast cancer were collected by core biopsy. The collected material was fixed in $10 \%$ formalin, embedded in paraffin, sectioned with $3-\mu \mathrm{m}$ thick and then stained with hematoxylin-eosin (H\&E). Then, immunohistochemistry assay was performed for 29 and 28 samples of IBC and LABC, respectively.

\section{3 | Tissue Microarray}

Tissue samples were obtained from the donor paraffin block with a manual puncher and arrayed in a matrix of six rows and three columns with up to 18 samples per recipient block. Liquid paraffin was gently added to melt the wax cylinders at $60^{\circ} \mathrm{C}$. Then, typical H\&E staining and immunohistochemistry assay were performed. Two independent observers who were unaware of clinicopathological data conducted the analysis. The following score criteria for tumor and stromal cells, adapted from the previous publication, ${ }^{14}$ were employed: zero expression (0), mild reaction $(1+)$, moderate reaction $(2+)$ and strong reaction (3+). Score analysis resulted in a high level of concordance (>90\%). Cases out of concordance were defined by consensus. The average value obtained for each sample was used to statistical analyzes. Slides were observed in an Olympus BX41 microscope.

\subsection{Immunohistochemistry assay}

Paraffin sections were dewaxed and rehydrated. Treatment with a 3\% $\mathrm{H}_{2} \mathrm{O}_{2}$ solution in methanol, for 10 min, was used to block endogenous peroxidase. An EnVision FLEX buffer target retrieval solution (Agilent Technologies, Santa Clara, CA, USA) in $96^{\circ} \mathrm{C}$ warm water, for $20 \mathrm{~min}$, was applied to unmask the antigens. Envision Flex Peroxidase-Blocking Reagent was utilized for $10 \mathrm{~min}$ to inhibit unspecified ground reactions. The slides were incubated with rabbit monoclonal antibodies anti-NF$\kappa$ B (Santa Cruz Biotechnology, Dallas, TX, USA; 1:200) or anti-IL-18 (Sigma-Aldrich, St. Louis, MA, USA, 1:400); liver and pancreas tumor samples were used as positive controls at 1:50 dillution. Negative controls in which no primary antibody was applied were included. Following primary antibody incubation, a secondary biotinylated antibody donkey anti-rabbit IgG (Agilent Technologies, Santa Clara, CA, USA) 1:100 was applied. Then, the streptavidin-coupled peroxidase complex (TS-125-HR; LabVision, Sao Paulo, Brazil) was used for $15 \mathrm{~min}$. An automated immunostainer (Agilent Technologies, Santa Clara, CA, USA) was utilized to process the reactions, and diaminobenzidine was used as the chromogen. The sections were counterstained with hematoxylin, dehydrated, diaphanized, mounted and analyzed.

\subsection{Statistical analysis}

Patient and tumor characteristics were tabulated and stratified by breast cancer type (IBC vs $\mathrm{LABC}$ ) and compared across groups with the chi-square test or Fisher's exact test when appropriate. For each crossing, the odds ratios with $95 \%$ of confidence interval were calculated. Overall survival was calculated from the time of diagnosis to the date of death or the time of last follow-up. The survival curves were estimated with the Kaplan-Meier method and the log-rank test was used to 
test for differences between groups. The curves were confronted with variables RE, RP, C-erbB2 and triple negative by log-rank test. In addition, we used a multivariate analysis by the Cox regression model to identify the prognosis factors that independently affect the survival of the patients. $P<0.05$ was considered significant. All data were analyzed using GraphPad Prism software version 6.01 and SPSS Statistics 22.0.

\section{3 | RESULTS}

\section{1 | Clinico-pathological characterization of IBC versus $L A B C$}

As shown in Table 1, there was no statistical difference $(P=0.324)$ between the IBC and $L A B C$ groups regarding the age of diagnosis. The mean age observed for the IBC and LABC groups was 49 and 51, respectively. The characteristics of tumor specimens are also shown in Table 1 . The histology analysis showed that most of the tumors were invasive ductal carcinoma (IDC), which represents $85.3 \%$ of IBC cases and $90 \%$ of $L A B C$. A similar distribution among histological grades was also observed, with approximately $90 \%$ of the IBC and LABC tumors classified as grades 2 and 3 . Regarding the hormone receptors expression (ER, PR, C-erbB2 and triple negative), no statistical difference between the two groups was found.

\section{2 | Overall survival}

The overall survival analysis showed that patients diagnosed with IBC showed a significant $(P<0.001)$ reduced survival when compared to patients with $L A B C$ (Figure 1). The odds ratio of two-year survival from diagnosis of patients with IBC was 14.4 ( $95 \%$ confidence interval 3.5 58.9; Table S1) compared to patients with LABC. For five-year survival, the odds ratio was 6.61 (95\% interval confidence 1.7-25.3; Table S1). Despite no difference concerning locoregional recurrence (data not shown), patients with IBC clearly survived less ( $45.5 \%$ of patients died in two years) than patients with LABC (20.4\%, $P=0.0002)$. Considering a five-year survival, $12.3 \%$ of patients with IBC are alive, whereas in the group of $L A B C$ this percentage was $37.6 \%$ (Figure 1 ).

\subsection{Overall survival according to the expression of tumor markers (ER, PR and C-erbB2) or triple negative patients}

Figure 2A shows that $L A B C$ patients positive for estrogen receptor $\left(E R^{+}\right)$have a higher survival compared to the $\mathrm{ER}^{+} \mathrm{IBC}$ group. We observed that $50 \%$ of $E R^{+} L A B C$ patients survived 4.4 years, whereas $\mathrm{ER}^{+} \mathrm{IBC}$ individuals survived only 2.3 years $(P=0.006)$. Regarding PR expression, no difference between the two populations was observed $(P>0.05$, Figure 2B). The contribution of C-erbB2 on patients' survival was also described. As observed in Figure $2 \mathrm{C}$, IBC patients that were negative to $\mathrm{C}$-erbB2 ( $\mathrm{C}$-erb2 ${ }^{-}$) showed a worse prognosis (2.1-year median survival) compared to C-erb2- LABC group (4.4-year survival, $P<0.001)$. Triple negative patients were also assessed. As
TAB LE 1 Clinical and pathological characterization of inflammatory breast cancer (IBC) vs noninflammatory locally advanced breast cancer (LABC)

\begin{tabular}{|c|c|c|c|c|}
\hline Characteristics & IBC & $\begin{array}{l}\text { LABC } \\
\text { (\%) }\end{array}$ & OR $(95 \% \mathrm{Cl})$ & $P$ value \\
\hline \multicolumn{5}{|l|}{$\begin{array}{l}\text { Age at diagnosis } \\
\text { (years) }\end{array}$} \\
\hline Up to 40 & $15(24.6)$ & $8(14.3)$ & $2.81(0.61-12.97)$ & $0.324^{a}$ \\
\hline $41-50$ & 19 (31.1) & 19 (33.9) & $1.50(0.36-6.18)$ & \\
\hline $51-60$ & $12(19.7)$ & $17(30.4)$ & $1.06(0.24-4.58)$ & \\
\hline $61-70$ & $11(18.0)$ & $6(10.7)$ & $2.75(0.55-13.75)$ & \\
\hline$>70$ & $4(6.6)$ & $6(10.7)$ & 1.00 & \\
\hline Unknown & 14 & 4 & - & \\
\hline \multicolumn{5}{|l|}{ Histological type } \\
\hline IDC & $64(85.3)$ & $54(90)$ & $1.77(0.28-11.04)$ & $0.299^{b}$ \\
\hline ILC & $2(2.7)$ & $3(5)$ & 1.00 & \\
\hline Others & $9(12)$ & $3(5)$ & $4.5(0.5-41.2)$ & \\
\hline Unknown & - & - & - & \\
\hline \multicolumn{5}{|c|}{ Histological grade } \\
\hline 1 & $2(5.7)$ & $3(9.1)$ & 1.00 & $0.861^{b}$ \\
\hline 2 & $16(45.7)$ & $15(45.5)$ & $1.6(0.23-10.95)$ & \\
\hline 3 & $17(48.6)$ & $15(45.5)$ & $1.7(0.25-11.59)$ & \\
\hline Unknown & 40 & 27 & - & \\
\hline \multicolumn{5}{|l|}{ Status ER } \\
\hline Positive & $28(49.1)$ & $27(48.2)$ & $1.04(0.5-2.17)$ & $1.000^{b}$ \\
\hline Negative & $29(50.9)$ & $29(51.8)$ & 1.00 & \\
\hline Unknown & 18 & 4 & - & \\
\hline \multicolumn{5}{|l|}{ Status PR } \\
\hline Positive & $22(40)$ & $24(43.6)$ & $0.86(0.40-1.83)$ & $0.846^{b}$ \\
\hline Negative & $33(60)$ & $31(56.4)$ & 1.00 & \\
\hline Unknown & 20 & 5 & - & \\
\hline \multicolumn{5}{|l|}{ Status C-erbB2 } \\
\hline Positive & $18(32.7)$ & $16(28.6)$ & $1.22(0.54-2.73)$ & $0.683^{b}$ \\
\hline Negative & $37(67.3)$ & 40 (71.4) & 1.00 & \\
\hline Unknown & 20 & 4 & - & \\
\hline \multicolumn{5}{|l|}{ Triple negative } \\
\hline Yes & $19(33.3)$ & $16(28.6)$ & $1.25(0.56-2.78)$ & $0.685^{b}$ \\
\hline No & $38(66.7)$ & 40 (71.4) & 1.00 & \\
\hline Unknown & 18 & 4 & - & \\
\hline
\end{tabular}

$\mathrm{Cl}$, confidence interval; C-erbB2, growth factor receptor human epidermal 2; ER, estrogen receptor; IDC, invasive ductal carcinomas; ILC, invasive lobular carcinomas; OR, odds ratio; PR, progesterone receptor.

${ }^{\text {a}}$ Fisher's exact test.

${ }^{\mathrm{b}} \mathrm{Chi}$-square test.

shown in Figure 2D, triple negative IBC patients also presented the worse prognosis (2-year survival) when compared to triple negative LABC group (3.4-year survival, $P=0.049$ ). In multivariate analysis, the independent prognosis factors affecting the survival of patients with breast cancer the histologic grade $3(P=0.021)$ showed a survival rate 1.001 worse and the triple negative immune staining $(P=0.002)$ with a survival rate 1.004 worse (Table S2). 


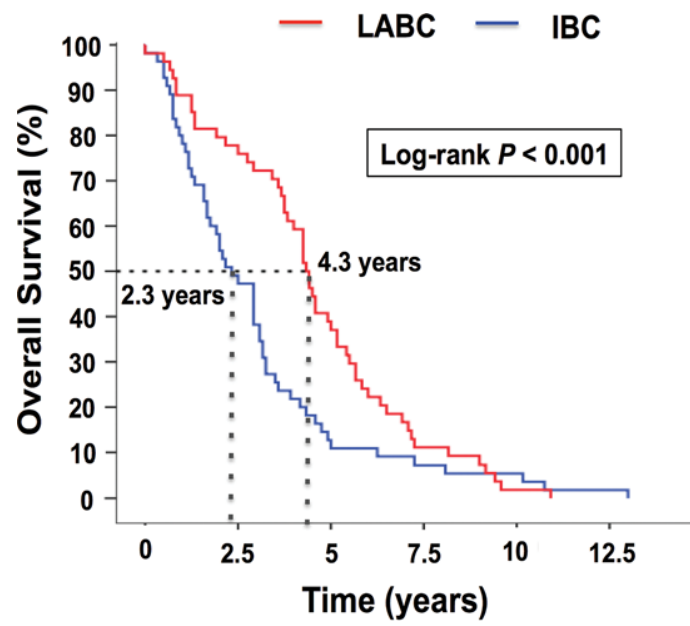

\begin{tabular}{lcccc}
\hline \multirow{2}{*}{ Groups } & $\begin{array}{c}\text { Survival } \\
\text { Mean }\end{array}$ & \multicolumn{2}{c}{ Survival (\%) } & P Value \\
\cline { 3 - 4 } & 5a & $10 \mathrm{a}$ & \\
\hline IBC & 2.3 & 12.3 & 2.7 & $<0.001$ \\
LABC & 4.3 & 33.6 & 3.3 & \\
\hline
\end{tabular}

FIGURE 1 Kaplan-Meier curve to overall survival of patients with inflammatory breast cancer (IBC) and noninflammatory locally advanced breast cancer (LABC). Kaplan-Meier method was applied to evaluate survival of patients the log-rank test was used to compare survival curves between groups [Colour figure can be viewed at wileyonlinelibrary.com]

\subsection{Immunohistochemistry to NF- $\kappa$ B and IL-18}

As shown in Table S3, positive immunostaining to NF- $\kappa \mathrm{B}$ (score 23) was observed in $46.4 \%$ of IBC cases and $36.3 \%$ of LABC group $(P>0.05)$. Depicts representative photomicrographs of immunostaining to NF- $\kappa$ B. The arrowheads indicate a strong immunoexpression of $\mathrm{NF}-\kappa \mathrm{B}$ in the tumor tissue (Figure S1). Furthermore, no association was found between the NF- $\kappa$ B expression with molecular markers (ER $\mathrm{PR}, \mathrm{C}$-erbB2 or triple negative tumors), neoadjuvant chemotherapy response, or overall survival in IBC and LABC (data not shown).

IL-18 immunostaining was also investigated (Tables S4 and S5). A stronger positivity (score $2-3$ ) was found in the stroma (27.6\% of samples) when compared to IL-18-positive tumor cells $(0 \%, P=0.004$; Table S3) in IBC group. Similar findings were observed in LABC group (50\% in the stroma vs $3.5 \%$ in the tumor, $P<0.001$; Table 4). However, when IL-18 immunostaining was compared in tumor (IBC: 0\% vs LABC: $3.5 \%, P>0.05$ ) or in stroma (IBC: $27.6 \%$ vs LABC: $50 \%, P=0.093$ ), no statistical differences were detected (Table S4). A representative photomicrograph is depicted in Figure 3. The arrows indicate the mild or moderated IL-18 expression in tumor cells and arrowheads shows the marked IL-18 expression in stromal cells. In addition, no statistical association between IL-18 expression with molecular markers (ER, PR, C-erbB2 or triple negative, $P>0.05$ ), overall survival (data not shown) or for neoadjuvant chemotherapy response $(P=0.061$; Table 2$)$ was detected for both breast cancer subtypes IBC and LABC.

\section{4 | DISCUSSION}

In this study was evaluated the expression of transcription factor NF$\kappa \mathrm{B}$ and IL-18 in tissue samples obtained from patients with IBC and LABC. These inflammatory markers were investigated for association with patients' prognosis and with the expression of breast cancer biomarkers (ER, PR and C-erbB2).

Demographic data analysis showed that there was no difference between the IBC and $\mathrm{LABC}$ regarding the prevalence of breast cancer subtypes according to age groups. The average age at diagnosis for IBC and LABC groups was 49 and 51 years old, respectively. However, younger women (up to 40 years old) seemed to be more affected by IBC than by $L A B C$. Such finding is in accordance with the literature, because the IBC seems to be more frequent in younger women, average age at diagnosis is 59 years old, when compared to 66 years old for other types of breast cancer. ${ }^{2}$ In a series of 74 patients with IBC reported by SEER (Surveillance, Epidemiology, and End Results) showed a mean age at diagnosis, which did not differ between the groups, although being higher than that observed in the present study, 57.2 years for IBC and 57.4 years for $L A B C .{ }^{15}$ These differences concerning the age at diagnosis might involve the population in study, genetic variability and environmental factors.

In regard to positivity for biomarkers (ER, PR and C-erbB2), no significant difference was observed between the two groups, which corroborates previously published data. ${ }^{15}$ However, it is worth to note that positivity to ER represented a positive prognostic factor to $L A B C$ group compared with IBC $\mathrm{ER}^{+}$tumors, because a significantly higher overall survival was found in that group when compared to the IBCER ${ }^{+}$ group. Data from the SEER with a series of 2237 patients diagnosed with IBC and 7985 cases of $\mathrm{LABC}$ showed that $\mathrm{ER}^{+}$-expressing LABC patients have a significantly higher survival ( 80 months) compared to patients IBC that express ER, which survive an average of 60 months. ${ }^{15}$ It is important to mention two aspects based on SEER study: (1) positivity to ER enhanced patients' survival of both $L A B C$ and IBC groups, which differed from the present study; (2) survival of North American patients are significantly higher than patients form this study. Such difference seems to rely on the time of diagnosis and access to primary health care. In Brazilian population, cancer diagnosis is commonly on advanced stage.

Here, we also found a similar C-erbB2 positivity for IBC and LABC (32.7\% and $28.6 \%$, respectively), which is in agreement with data observed in the study from the Royal Marsden Hospital in London that showed a $38 \%$ of C-erbB2 expression in IBC patients, ${ }^{4}$ and the study from the MD Anderson Cancer Center identified $44.3 \%$ of IBC samples also positive to C-erbB2. ${ }^{16}$ The absence of oncoprotein C-erbB2 expression has a significant impact on patient survival in IBC group compared to LABC. Interestingly, other studies have suggested that lack of C-erbB2 along with ER and PR negativity also significantly worsened the survival of patients in the IBC group when compared to LABC group. ${ }^{17,18}$

We also observed a mean survival of only 25 months ( 2.3 years) in IBC group, but a 51-month survival (4.3 years) in LABC group. The worse survival of IBC patients compared to $\angle A B C$ individuals is also reported in previous studies. ${ }^{4,19}$ Accordingly, Sutherland et al. ${ }^{4}$ 
A

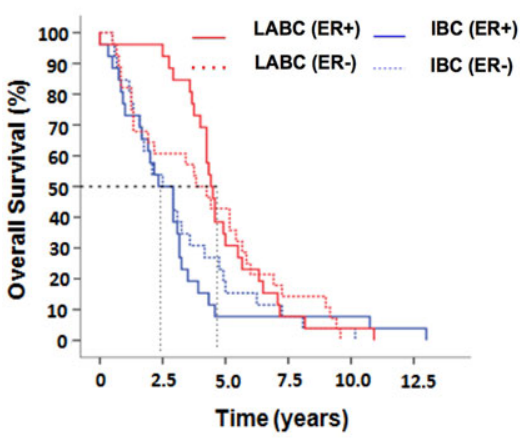

\begin{tabular}{cccc}
\hline \multicolumn{2}{c}{ Status ER } & $\begin{array}{c}\text { Survival } \\
\text { Median }\end{array}$ & $P$ value \\
\hline \multirow{2}{*}{ ER+ } & IBC & 2.3 & 0.006 \\
& LABC & $4.4^{*}$ & \\
ER- & IBC & 2.5 & 0.292 \\
\hline
\end{tabular}

C

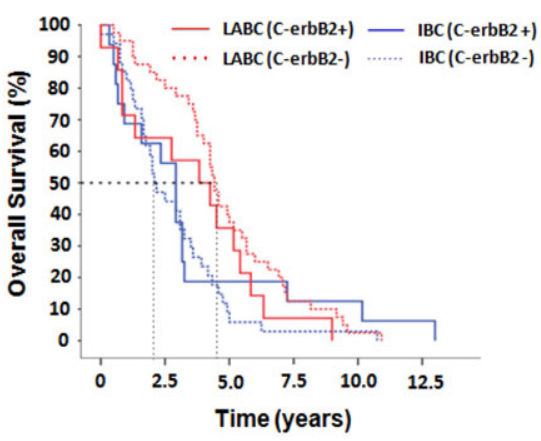

\begin{tabular}{|c|c|c|c|}
\hline Status & erbB2 & $\begin{array}{l}\text { Survival } \\
\text { Median }\end{array}$ & $P$ value \\
\hline C-erbB2+ & $\begin{array}{c}\text { IBC } \\
\text { LABC }\end{array}$ & $\begin{array}{l}2.9 \\
3.8\end{array}$ & 0.934 \\
\hline C-erbB2- & $\begin{array}{c}\text { IBC } \\
\text { LABC }\end{array}$ & $\begin{array}{l}2.1 \\
4.4^{*}\end{array}$ & $<0.001$ \\
\hline
\end{tabular}

B

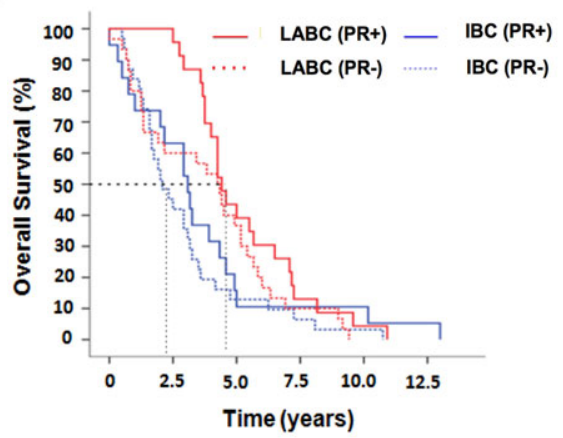

\begin{tabular}{cccc}
\hline \multicolumn{2}{c}{ Status PR } & $\begin{array}{c}\text { Survival } \\
\text { Median }\end{array}$ & $P$ value \\
\hline PR+ & IBC & 3.1 & 0.137 \\
LABC & 4.4 & \\
PR- & IBC & 2.1 & 0.193 \\
\hline & LABC & 4.3 & \\
\hline
\end{tabular}

D

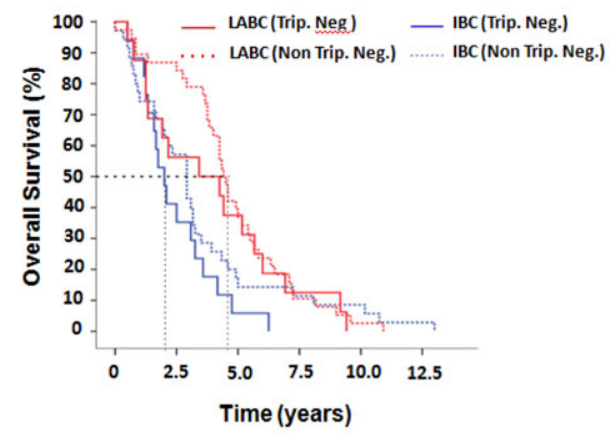

\begin{tabular}{|c|c|c|c|}
\hline \multicolumn{2}{|c|}{ Status Trip. Neg. } & \multirow{2}{*}{$\begin{array}{c}\begin{array}{c}\text { Survival } \\
\text { Median }\end{array} \\
2.0 \\
3.4^{\star}\end{array}$} & \multirow{2}{*}{$\frac{P \text { value }}{0.049}$} \\
\hline Trip. Neg. & $\begin{array}{l}\text { IBC } \\
\text { LABC }\end{array}$ & & \\
\hline Non Trip. Neg. & $\begin{array}{c}\text { IBC } \\
\text { LABC }\end{array}$ & $\begin{array}{l}2.9 \\
4.4\end{array}$ & 0.105 \\
\hline
\end{tabular}

FIGURE 2 Kaplan-Meier curves to overall survival of patients with inflammatory breast cancer (IBC) and noninflammatory locally advanced breast cancer ( $L A B C$ ) stratified by biological breast cancer markers (ER, PR and C-erbB-2). (A) Patients IBC or LABC positive or negative to estrogen receptor (ER). (B) Patients IBC or LABC positive or negative to progesterone receptor (PR). (C) Patients IBC or LABC positive or negative to C-erbB2 receptor (C-erbB2). (D) Patients IBC or LABC triple negative (Trip. Neg.) or not. Kaplan-Meier method was applied to evaluate survival of patients the log-rank test was used to compare survival curves between groups [Colour figure can be viewed at wileyonlinelibrary.com]

IBC

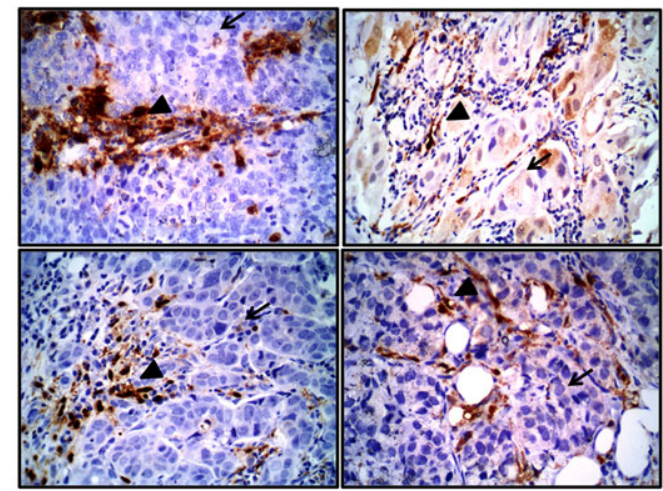

FIGURE 3 Immunostaining for IL-18 in inflammatory breast cancer (IBC) and noninflammatory locally advanced breast cancer (LABC). The arrows indicate the mild or moderated IL-18 expression in tumor cells and arrowheads shows the marked IL-18 expression in stromal cells. Representative photomicrographs, increase of 400x. [Colour figure can be viewed at wileyonlinelibrary.com] observed a 3.3-year survival for IBC patients. The study of Low et al. ${ }^{19}$ reported similar findings (a 3.8-year survival for IBC and 5.8 years for $\mathrm{LABC}$ ). Despite the differences in time of survival, the outcome is quite identical whatever the population.

Taking into account the aforementioned information, the underlying pathology of IBC is likely different of $L A B C$, which is probably unrelated to expression of ER, PR and C-erbB2. Therefore, the search for new biomarkers opens the perspective for potential therapeutic targets for breast cancer subtypes. Then, we evaluated the expression of $\mathrm{NF}-\kappa \mathrm{B}$ and IL-18 in tumors of IBC and LABC.

However, in spite of the broad description of the important role of $\mathrm{NF}-\kappa \mathrm{B}$ in the initiation and progression of breast cancer ${ }^{20}$ and the positive association of this transcription factor with C-erbB2, but negative with ER expression, ${ }^{21}$ we did not identify any association between these variables and prognostic factors (ER, PR and CerbB2), whatever the type of breast cancer investigated. However, we observed a high IL-18-positivity in stromal cells in both IBC and $\mathrm{LABC}$. The literature reported that IL-18 is expressed in tumor and 
TA B LE 2 Association of IL-18 expression with the clinical neoadjuvant chemotherapy response

\begin{tabular}{lccc} 
Clinical response to neoadjuvant chemotherapy & \multicolumn{2}{c}{ IL-18 } & OR (95\% CI) \\
\cline { 2 - 4 } Complete response & Negative (0-1) & Positive (2-3) & $0.5(0.05-5.51)$ \\
Partial response & $3(11.1 \%)$ & $6(30 \%)$ & $1.33(0.16-10.87)$ \\
\hline Stable disease & $16(59.3 \%)$ & $12(60 \%)$ & 1.00 \\
\hline Disease progression & $2(7.4 \%)$ & $2(10 \%)$ & $13(0.4-377.8)$ \\
\hline
\end{tabular}

$\mathrm{Cl}$, confidence interval; OR, odds ratio.

${ }^{\text {a}}$ Fisher's exact test, invasive ductal.

tumor microenvironment. ${ }^{13}$ In our opinion, the presence of IL-18 in the stroma might have an important role in the activation of an inflammatory antitumor response. Despite the lack of improved survival, other factors together with IL-18 seem to be necessary to overcome tumor immunosurveillance escape. The use of chemotherapeutic agents is known to activate antitumor immune response. ${ }^{22}$ As reviewed by Zitvogel et al. chemotherapy can mediate a multipronged immunostimulatory effect, thereby reinstating anticancer immunosurveillance, increasing the antigenicity of malignant cells, improving their immunogenicity, and augmenting their susceptibility to immune attacks. $^{22}$ Interestingly, we found a likely enhanced complete neoadjuvant response $(P=0.061)$ in association with IL-18. Then, the precise contribution of IL-18 to improvement of chemotherapy effectiveness merits further investigation.

Apparently, the aggressiveness of the IBC seem also involve other immune system mechanisms, such as, IL-10, TGF- $\beta$ (Transforming Growth Factor Beta), regulatory T cells and M2 macrophages. These cells and mediators are responsible to regulate immune response tumor escape, leading to disease progression and metastasis. ${ }^{6}$ Therefore, understanding the role of these mediators could help to identify specific markers for the IBC, what could explain the faster tumor progression and the shorter disease-free interval.

Immunity is now a major target for treating cancer. This study attempted to show IL-18 and NF- $\kappa$ B as potential prognostic markers of neoadjuvant chemotherapy response in patients with IBC and LABC. The lack of positive correlation might be related to small sample size or the population of study. Despite no statistical significance for the markers studied, the present research might be an important step for effective clinical management of breast cancer, because the role of other inflammatory markers that regulate immune function must be investigated. Additional studies are highly encouraged also with other ethnic groups.

\section{5 | CONCLUSION}

In conclusion, the chemotherapy neoadjuvant complete response seems to be partially associated with IL-18 expression in stroma of IBC and $L A B C$.

\section{ACKNOWLEDGMENTS}

This study is dedicated to the loving memory of Prof. Dr. Ronaldo Albuquerque Ribeiro (in memoriam). R.C.P. Lima-Júnior received a research
Grant from CNPq (Conselho Nacional de Desenvolvimento Científico e Tecnológico), Grant Nos.: 307143/2014-7 and 458872/20148. This work was also supported by Grants from CAPES (Fundação Coordenação de Aperfeiçoamento de Pessoal de Nível Superior, Grant CAPES-PROEX 2862/2013) and FUNCAP (Fundação Cearense de Apoio ao Desenvolvimento Científico, Grant PRONEX PR2-010100054.01.00/15). de Apoio ao Desenvolvimento Científico.

\section{CONFLICTS OF INTEREST}

The authors indicate that they have no potential conflicts of interest.

\section{REFERENCES}

1. Torre L, Bray F, Siegel RL, et al. Global Cancer Statistics. CA Cancer J Clin 2015;65:87-108.

2. Taghian A, El-Ghamry M, Merajver SD. Overview of the treatment of newly diagnosed, non-metastatic breast câncer. Up to date [online], Available at: http://www.uptodate.com/ contents/overview-of-the-treatment-of-newly-diagnosed-non-metas tatic-breast-cancer?source=search_result\&search=cancer+de+mam a\&selectedTitle=1 150. [Accessed on 16 December 2016].

3. Yamauchi H, Woodward WA, Valero V, et al. Inflammatory breast cancer: what we know and what we need to learn. Oncologist 2012;17:891-899.

4. Sutherland S, Ashley S, Walsh G, et al. Inflammatory breast cancer - The Royal Marsden Hospital experience. Cancer 2010;116:28152820.

5. Merajver S, Sabel MS. Inflammatory breast cancer. In: Harris JR, Lippman ME, Morrow M, Osborne CK. Diseases of the breast. 3rd ed. Philadelphia: Lippincott Williams and Wilkins, 2004.

6. Hanahan, Weinberg. Hallmarks of cancer: the next generation. Cell 2011;144: 646-674.

7. Giuliani C, Napolitano G, Bucci I, et al. NF- $\kappa$ B transcription factor: role in the pathogenesis of inflammatory, autoimmune, and neoplastic diseases and therapy implications. Clin Ter. 2001;152:249-253.

8. Biswas DK, Shi Q, Baily S, et al. NF- $\kappa$ B activation in human breast cancer specimens and its role in cell proliferation and apoptosis. Proc Natl Acad Sci. 2004;6:10137-10142.

9. Baker RG, Hayden MS, Ghosh S. NF- $\kappa$ B, inflammation, and metabolic disease. Cell Metabolism 2011;13:11-22.

10. Chariot KSA. NF- $\kappa$ B, stem cells and breast cancer: the links get stronger. Breast Cancer Research 2011;13:https:// doi.org/10.1186/bcr2886.

11. Liu L, Ke Y, Jiang X, et al. Lipopolysaccharide activates ERK-PARP-1RelA pathway and promotes nuclear factor $-\kappa$ B transcription in murine macrophages. Hum Immunol. 2012;73:439-447. 
12. Dinarello CA. Interleukin 1 and interleukin 18 as mediators of inflammation and the aging process. Am J Clin Nutr. 2006;83:447-455.

13. Srabović N, Mujagić Z, Mujanović-Mustedanagić J, et al. Interleukin 18 expression in the primary breast cancer tumour tissue. Off Publ Med Assoc Zenica-Doboj Canton Bosnia and Herzegovina 2011;8:109-115.

14. Meteoglu I, Erdogdu IH, Meydan N, et al. NF-KappaB expression correlates with apoptosis and angiogenesis in clear cell renal cell carcinoma tissues. J Exp Clin Cancer Res. 2008;27:53-62.

15. Anderson WF, Chu KC, Chang S. Inflammatory breast carcinoma and noninflammatory locally advanced breast carcinoma: distinct clinicopathologic entities? J Clin Oncol. 2003;21: 2254-2259.

16. Robertson FM, Bondy M, Yang W, et al. Inflammatory Breast Cancer: The Disease, the Biology, the Treatment. CA Caner J Clin. 2010;60: 351-375.

17. Dawood S, Broglio K, Gong $Y$ et al. Prognostic significance of HER-2 status in women with inflammatory breast cancer. Cancer 2008;112:1905-1911.

18. Gianni L, Eiermann W, Semiglazov V et al. Neoadjuvant chemotherapy with trastuzumab followed by adjuvant trastuzumab versus neoadjuvant chemotherapy alone, in patients with HER2-positive locally advanced breast cancer (the NOAH trial): a randomised controlled superiority trial with a parallel HER2-negative cohort. Lancet 2010;375:377-384

19. Low JA, Berman AW, Steinberg SM, et al. Long-term follow-up for locally advanced and inflammatory breast cancer patients treated with multimodality therapy. J Clin Oncol. 2004;22:4067-4074.

20. Zubair A, Frieri M. Role of nuclear factor-kB in breast and colorectal cancer. Curr Allergy Asthma Rep. 2013;13:44-49.

21. Ahmed KM, Cao N, Li JJ, et al. HER-2 and NF-kappaB as the targets for therapy-resistant breast cancer. Anticancer Res. 2006;26:4235-4243.

22. Zitvogel L, Galluzzi L, Smyth MJ, et al. Mechanism of action of conventional and targeted anticancer therapies: reinstating immunosurveillance. Immunity 2013;39:74-88.

\section{SUPPORTING INFORMATION}

Additional Supporting Information may be found online in the supporting information tab for this article.

How to cite this article: Aguiar MAN, Wanderley CWS, Nobre LMS, et al. Interleukin-18 (IL-18) is equally expressed in inflammatory breast cancer and noninflammatory locally advanced breast cancer: a possible association with chemotherapy response. Asia-Pac J Clin Oncol. 2017;0:1-7. https://doi.org/10.1111/ajco.12722 